\title{
Delayed Diagnosis of Meralgia Paresthetica: A Case Report
}

\author{
Woo-chul Cho ${ }^{1}$, Byung-chul Son ${ }^{1,2}$ \\ ${ }^{1}$ Department of Neurosurgery, Seoul St. Mary's Hospital, College of Medicine, The Catholic University of Korea, Seoul, \\ ${ }^{2}$ Catholic Neuroscience Institute, College of Medicine, The Catholic University of Korea, Seoul, Republic of Korea
}

Corresponding author:

Byung-chul Son

Department of Neurosurgery,

Seoul St. Mary's Hospital,

Catholic Neuroscience Institute,

College of Medicine, The

Catholic University of Korea,

222 Banpo-daero, Seocho-gu,

Seoul 06591, Republic of Korea

Tel: +82-2-2258-6122

Fax: +82-2-594-4248

E-mail: sbc@catholic.ac.kr

Received: May 26, 2018

Revised: June 14, 2018

Accepted: June 19, 2018
Meralgia paresthetica (MP, or Bernhardt-Roth syndrome) is a painful mononeuropathy resulting from the compression of the lateral femoral cutaneous nerve (LFCN) as it crosses between the anterior superior iliac spine and the inguinal ligament to enter the thigh. Although the diagnosis of MP is straightforward based on its typical presentation, other neuropathies such as lumbar disc herniation or stenosis may mimic MP and complicate the diagnosis. We report a case of delayed diagnosis of MP due to concomitant lumbar disc herniation. A 49-year-old female patient presented with a 2-year history of gradually worsening pain and paresthesias in her left anterior thigh and left buttock. Magnetic resonance imaging of the lumbar spine revealed disc herniation at the level of L4/5. The diagnosis of MP was made after relief from buttock pain using repeated epidural blocks. Although MP was diagnosed late, decompression of the LFCN resulted in immediate and permanent relief of chronic anterior thigh pain. Unexplained, chronic anterolateral thigh pain and paresthesias warrant careful investigation for the possibility of MP.

Key Words: Femoral nerve; Nerve compression syndromes; Pain

\section{INTRODUCTION}

Meralgia paresthetica (MP) is a painful mononeuropathy of the lateral femoral cutaneous nerve (LFCN) ${ }^{2,3)}$. It occurs following entrapment of LFCN as it exits the pelvis and pierces the inguinal ligament ${ }^{2)}$. It is usually reported in patients sustaining trauma to the inguinal ligament, individuals assuming squatting postures for prolonged periods, and in cases involving leg-length discrepancies, with the pelvic-tilt stretching the fascia lata and consequently the nerve against the entrapment point ${ }^{2,6)}$. Although pregnancy and obesity-associated pannus are the most common scenarios, tight-clothing, belt holsters, iliac bone graft harvest, and entrapment from surgical scarring have all been implicated $^{2,3,6)}$. Pain and paresthesia, which may progress to numbness of the upper lateral thigh, are the key features of $\mathrm{MP}^{2)}$.

In the classic form of MP, the symptoms are fairly stereotypical and the clinical diagnosis is fairly straightforward ${ }^{7,9)}$. However, a diagnosis of MP is not always straightforward in clinical practice ${ }^{7,9)}$. This condition can be easily confused with lumbosacral radicular pain in a small group of patients ${ }^{7,9)}$. In the current case, the diagnosis of MP was fairly delayed by coexisting symptoms of lumbar disc herniation.

\section{CASE REPORT}

A 49-year-old right-handed female patient presented with a 2-year history of gradually worsening pain and paresthesias, in her left buttock, anterior thigh, and leg. Two years ago, the patient was afflicted with sudden pain in her left lateral buttock and posterior thigh without any precipitating event. Since then, left-sided buttock and leg radiating pain occurred intermittently after a prolonged period of standing and working once or twice a month. The pain was relieved with over-the-count medications and bed rest within 2 weeks. One year prior to the recent presentation, sudden, aching pain and paresthesia developed in her left groin and radiated to anterior and lateral thigh. The pain in her left groin, buttock and lateral and posterior thigh developed during standing for more than $30 \mathrm{~min}$. She was diagnosed with lumbar disc herniation at L4/5 after magnetic resonance imaging (MRI) of the lumbar spine. She was treated conservatively with regular medication and physical therapy. However, her pain in the left buttock and posterolateral thigh failed to improve and worsened gradually. She visited our outpatient clinic for further evaluation and treatment.

She complained of recurrent episodes involving her left buttock and radiating pain in her left anterior thigh. Back and anterior thigh pain prevented the patient from work for 1 week. Physical examination revealed no tenderness or limitation of back motion. No objective signs of motor or sensory abnormality was detected in the physical examinations. The pain in the buttock was described as mainly dull aching with moderate severity (4-5/10 on numerical rating scale; range, 0-10). It was 
aggravated with prolonged standing or walking for more than $20 \mathrm{~min}$. Straight leg raise was mildly restricted in the left leg (80/60). Deep tendon reflex was normal. Laboratory examinations were normal, including erythrocyte sedimentation rate and C-reactive protein. MRI of the lumbar spine revealed a left-sided herniated disc at the level of L4/5 (Fig. 1A). Lumbar epidural blocks were performed twice considering the possibility of lumbar disc herniation. Two weeks after epidural blocks, the patient reported pain improvement of left buttock and posterior thigh. However, her pain and paresthesia in her anterior thigh did not improve and, even, deteriorated despite pain relief in the buttock. Additional history and physical examination were considered. Indeed, the patient had already reported pain and paresthesia in her left anterior thigh in addition to posterior thigh pain during the first interview at the outpatient clinic. However, we failed to understand the importance of anterolateral thigh pain.

Her groin pain originated in her left inguinal area and radiated to anterior thigh. It also radiated to left lower quadrant of the
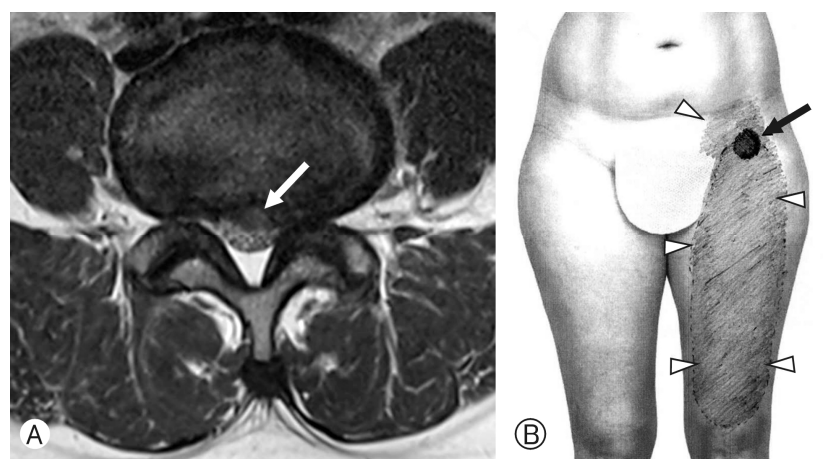

Fig. 1. Presentations of anterior thigh pain in meralgia paresthetica. (A) A T2 axial (right) and T2 sagittal (left) images in the magnetic resonance imaging of lumbar spine. Lumbar disc herniation (arrows) was found at the left side of the L4/5. (B) Distribution of anterior thigh pain. Tender point (black arrow) was found just medial to the anterior superior iliac spine. Radiating from the tender point, paresthesia and pain spread to lower abdomen and anterior thigh. abdomen. A mild tenderness was noted in her left inguinal area around left anterior superior iliac spine (ASIS) (Fig. 1B). Tinel's sign was positive. Burning dysesthesia and paresthesia in her anterior thigh were described. No objective signs of sensory abnormality was detected. A diagnostic block of the LFCN with $5 \mathrm{~mL}$ of $1 \%$ lidocaine was effective for $6 \mathrm{hr}$. Decompression of the LFCN was proposed due to chronicity and medical intractability of MP. Three-dimensional computed tomographic (CT) scan and MRI of the pelvis showed no mass lesion or anatomical abnormality in the left inguinal area.

An approximately 6-cm skin incision was created below the ASIS over the course of left inguinal ligament, down to the fascia lata. The LFCN was carefully identified from the Sartorius muscle and decompressed distally by opening the fascia lata (Fig. $2 \mathrm{~A})^{2,3)}$. It was then followed proximally, and the entrapment point was found in the inguinal ligament and tendinous arc from the iliac fascia (Fig. 2B). The portion of the inguinal ligament and tendinous arc of the iliac fascia abutting the LFCN were divided, freeing the LFCN (Fig. 2C). Immediately following decompression of the left LFCN, no repeated attacks of pain in the left inguinal and anterior thigh occurred. No sensory abnormality, pain or paresthesia in her left groin and thigh was reported until 12 month follow-up.

\section{DISCUSSION}

\section{MP, a Painful Mononeuropathy of LFCN}

Bernhardt-Roth syndrome, better known as MP, was first described in 18783), and the first decompression was performed by Harvey Cushing in $1900^{2,5)}$. It occurs following entrapment of the LFCN as it exits the pelvis and penetrates the inguinal ligament. Multiple causes of MP, include pregnancy, external compression such as obesity-associated panniculus, tight clothing, belts or gun holsters, diabetes, hypothyroidism, iliac bone graft harvesting, trauma and surgical scarring ${ }^{2,3,6,7)}$. Symptoms include pain, paresthesias, hypesthesias, or dysesthesias
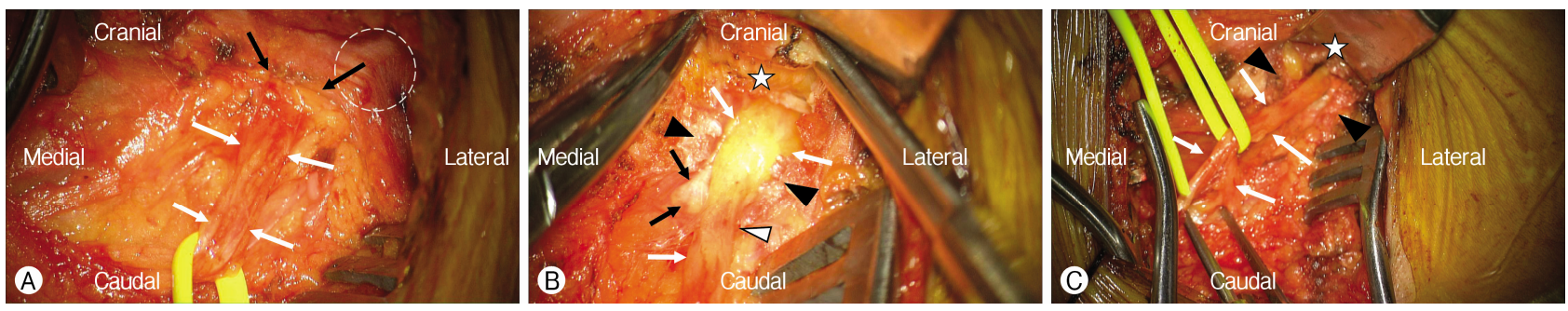

Fig. 2. Decompression of the lateral femoral cutaneous nerve (LFCN): intraoperative findings. (A) Dissection of the LFCN (white arrows) from the sartorius muscle and distal decompression by opening the fascia lata. The LFCN was followed proximally under the inguinal ligament (black arrows). White circle indicates anterior superior iliac spine. (B) The inguinal ligament (black arrowheads) is sectioned, including the tendinous arch of the iliac fascia (white star) during proximal decompression of the LFCN (white arrows). White arrowhead indicates area of maximal constriction of the LFCN. Note the underlying part of the inguinal ligament (black arrows) still abutting the LFCN. (C) An intraoperative image showing decompression of LFCN both distally and proximally (white star). The medial and lateral branches (white arrows) of the LFCN over the sartorius muscle are notable. 
localized to the anterolateral hip, anterolateral thigh, and distally to the anterolateral knee ${ }^{3)}$. Signs of MP include tenderness over the LFCN next to the ASIS and a positive Tinel's sign ${ }^{2,3}$. The scratch-collapse test is often positive at the exit point of the nerve just medial to the ASIS ${ }^{2)}$. Electrodiagnostic test may identify attenuated sensory nerve action potentials or reduced sensory nerve conduction velocities ${ }^{2}$. Conservative management of MP involves avoidance of exposure to the offending sources whenever possible, i.e., by weight loss and avoidance of constricting garments or accessories in addition to nonsteroidal anti-inflammatory drugs, heat, and physical therapy. The majority of cases are successfully treated conservatively, and surgical treatment is reserved for those patients with refractory symptoms.

\section{Surgical Anatomy}

LFCN is composed of fibers from the posterior divisions of the $\mathrm{L} 2$ and $\mathrm{L} 3$ nerve roots. It traverses the psoas muscle and runs across the surface of the iliacus muscle. After piercing the fascia of the iliacus muscle, it approaches the inguinal ligament. Traditionally, the nerve exits a triangle constituted by the ASIS externally, the inguinal ligament superiorly, and the fascia of the Sartorius inferiorly ${ }^{2}$. However, several types of anatomical variations of the LFCN have been described ${ }^{1-3,9)}$. Aszmann et al. ${ }^{1)}$ identified five variants of the LFCN as it exits the pelvis. They suggested that the three variations are most susceptible to mechanical injury: posterior to the ASIS and across the iliac crest (type A); anterior to the ASIS and superficial to the origin of the sartorius muscle but within the substance of the inguinal ligament (type B); or medial to the ASIS, ensheathed in the tendinous origin of the Sartorius muscle (type C). Multiple branches of the LFCN were found as well. In line with this study, Ducic et al. ${ }^{3)}$ reported that an abnormal position of the LFCN was found in $35(73 \%)$ of 48 cases of neurolysis and decompression surgeries of the LFCN. According to Aszmann et al.'s report ${ }^{1}$, the current case belongs to type B.

\section{Differential Diagnosis}

Classically, MP is caused by compression of the LFCN at the site where it exits the pelvis, but other neuropathies may mimic $\mathrm{MP}^{3)}$. The differential diagnosis for anterolateral thigh pain and paresthesia is varied and includes lumbar radiculopathy; lumbar facet syndrome; increased retroperitoneal pressure resulting from tumor, hemorrhage, or abscess; mononeuritis multiplex; diabetic lumbosacral plexopathy; and lumbar spondylolysis an spondylolisthesis ${ }^{3}$. Spinal imaging is essential to exclude possible spinal etiology in suspected patients with MP. The most likely pathology mimicking MP is lumbar radiculopathy ${ }^{3,4,11)}$. MP yields no motor deficit as it is a purely sensory nerve with no lumbar pain, sciatic notch tenderness, or positive straight-leg raising sign ${ }^{3,11}$. Further, pain or presence of a positive Tinel's sign over the ASIS additionally support the diagnosis of $\mathrm{MP}^{3,4,11)}$. Injection of 1 percent lidocaine into the region of the tenderness provides relief from the paresthesia.

\section{Surgical Techniques and Results}

Surgical results of decompression of the LFCN in MP vary significantly, from 60 to $95 \%{ }^{2}$. The most successful series focused increasingly on the anatomical variation ${ }^{1-3,8)}$. Although van Eerten et al. ${ }^{10)}$ found $\mathrm{LFCN}$ resection superior to neurolysis, neurolysis is mostly recommend as a preferred treatment ${ }^{2,3}$.

Patients with obesity and long-standing neuropathies, as well those with multiple nerves involved or less clearly defined distributions, show poor outcome with neurolysis and decompression $^{2,8}$. Therefore, the importance of timely diagnosis and surgical decompression in cases refractory to medical treatment cannot be overstated. Decompression of the LFCN releases the trapped nerve at the inguinal ligament. However, it is imperative to explore more proximally and release the LFCN from the fascia of the internal oblique muscle and other deep abdominal musculature, as well distally toward the deep thigh fascia $^{3)}$.

\section{CONCLUSION}

The authors report a rare occurrence of MP associated with lumbar disc herniation. The diagnosis of MP is typically delayed by the coexistence of symptoms of lumbar disc herniation in addition to MP. Complaints of pain and paresthesia in the anterior and lateral thigh warrant investigation for possible $\mathrm{MP}$, a rare painful mononeuropathy involving the LFCN.

\section{CONFLICT OF INTEREST}

No potential conflict of interest relevant to this article was reported.

\section{REFERENCES}

1. Aszmann OC, Dellon ES, Dellon AL: Anatomical course of the lateral femoral cutaneous nerve and its susceptibility to compression and injury. Plast Reconstr Surg 100:600-604, 1997

2. Boyd KUB, J M. Injury and compression neuropathy in the lower extremity. In: Mackinnon SE, Yee A eds. Nerve surgery. New York, NY: Thieme, 2015, pp338-390

3. Ducic I, Dellon AL, Taylor NS: Decompression of the lateral femoral cutaneous nerve in the treatment of meralgia paresthetica. J Reconstr Microsurg 22:113-118, 2006

4. Lee $\mathrm{CH}$, Dellon AL: Surgical management of groin pain of neural origin. J Am Coll Surg 191:137-142, 2000

5. McCrory P, Bell S: Nerve entrapment syndromes as a cause 
of pain in the hip, groin and buttock. Sports Med 27:261-274, 1999

6. Morelli V, Weaver V: Groin injuries and groin pain in athletes: part 1. Prim Care 32:163-183, 2005

7. Nouraei SA, Anand B, Spink G, O'Neill KS: A novel approach to the diagnosis and management of meralgia paresthetica. Neurosurgery 60:696-700, 2007

8. Siu TL, Chandran KN: Neurolysis for meralgia paresthetica: an operative series of 45 cases. Surg Neurol 63:19-23, 2005
9. Son BC, Kim DR, Kim IS, Hong JT, Sung JH, Lee SW: Neurolysis for meralgia paresthetica. J Korean Neurosurg Soc 51:363366, 2012

10. van Eerten PV, Polder TW, Broere CA: Operative treatment of meralgia paresthetica: transection versus neurolysis. Neurosurgery 37:63-65, 1995

11. Williams PH, Trzil KP: Management of meralgia paresthetica. J Neurosurg 74:76-80, 1991 